\title{
Effects of Concomitant Administration of a Dipeptidyl Peptidase-4 Inhibitor in Japanese Patients with Type 2 Diabetes Showing Relatively Good Glycemic Control Under Treatment with a Sodium Glucose Co-Transporter 2 Inhibitor
}

\author{
Authors \\ Affiliations \\ 1 Research Center of Health, Physical Fitness and Sports, \\ Nagoya University, Nagoya, Japan \\ 2 Vascular Center, Sanno Medical Center, Tokyo, Japan \\ 3 Okinaka Memorial Institute for Medical Research, Tokyo, \\ Japan
}

Masataka Kusunoki ${ }^{1}$, Yukie Natsume ${ }^{1}$, Tetsuro Miyata ${ }^{2}$, Kazuhiko Tsutsumi ${ }^{3}$, Yoshiharu Oshida ${ }^{1}$

Key words

monotherapy, concomitant therapy, HbA1c, HDL cholesterol

received 26.06.2017

accepted 28.02.2018

\author{
Bibliography \\ DOI https://doi.org/10.1055/a-0585-0145 \\ Published online: 2.7.2018 \\ Drug Res 2018; 68: 704-709 \\ (c) Georg Thieme Verlag KG Stuttgart · New York \\ ISSN 2194-9379
}

\section{Correspondence}

Masataka Kusunoki

Research Center of Health

Physical Fitness and Sports

Nagoya University

Furo-cho, Chikusa-ku

Nagoya 464-8601

Japan

Tel.: + 81/52/789 3946, Fax: + 81/52/7893957

info@tonyo.jp

\begin{abstract}
We conducted this study to determine whether additional administration of a dipeptidyl peptidase-4 (DPP-4) inhibitor might provide further improvement of the glycemic control in Japanese type 2 diabetes patients showing relatively good glycemic control under treatment with a sodium glucose co-transporter 2 (SGLT2) inhibitor. Five SGLT2 inhibitor (luseogliflozin, dapagliflozin, tofogliflozin, empagliflozin and canagliflozin) preparations and five DPP-4 inhibitor (sitagliptin, vildagliptin, alogliptin, anagliptin and linagliptin) preparations were used. The results showed that monotherapy with SGLT2 inhibitor produced significant decreases of the body weight and BMI, hemoglobin A1c (HbA1c) also decreased, but not to a significant extent. However, decreases of the serum aspartate aminotransferase (AST), alanine aminotransferase (ALT), $\gamma$-glutamyltransferase ( $\gamma$-GTP) and uric acid were observed in this group. On the other hand, in type 2 diabetes patients treated concomitantly with a DPP-4 inhibitor and SGLT2 inhibitor, significant decrease of the HbA1c was observed, indicating the favorable effect of the concomitant therapy. The body weight and BMI decreased. As for the serum lipid profile, elevation of the serum HDL-cholesterol (HDL-C) was observed. Furthermore, AST, ALT, Y-GTP and uric acid decreased in the combined treatment group. Then, the therapeutic responses to concurrent administration with SGLT2 inhibitor of each of the 5 individual DPP-4 inhibitors used in this study were analyzed. The results showed that concomitant administration of sitagliptin, a DPP-4 inhibitor, with the SGLT2 inhibitor yielded the best results in terms of the lowering of the $\mathrm{HbA} 1 \mathrm{c}$ and improvement of the serum lipid profile.
\end{abstract}

\section{Introduction}

Concurrent treatment with two or more hypoglycemic agents with differing mechanisms of action may be useful in type 2 diabetes mellitus patients showing inadequate glycemic control under treatment with one drug alone. This study was designed to determine whether concurrent administration of a dipeptidyl peptidase- 4 (DPP-4) inhibitor might yield further improvement of the glycemic control in Japanese type 2 diabetes mellitus patients receiving a selective inhibitor of sodium glucose co-transporter-2 (SGLT2 inhib- itor). SGLT2 is a transporter protein expressed in the proximal renal tubules that mediates the reabsorption of approximately $90 \%$ of all the glucose filtered through the glomeruli [1,2]. SGLT2 inhibitors have been shown to exert their hypoglycemic effect by causing selective inhibition of SGLT2 in the kidneys, thereby facilitating urinary excretion of excessive circulating glucose [3]. In addition to their hypoglycemic effect, SGLT2 inhibitors have also been reported to reduce the body weight, lower the blood pressure, and improve lipid metabolism and uric acid metabolism [4]. In a previ- 
ously reported trial, treatment with a SGLT2 inhibitor, as compared to placebo, significantly suppressed the occurrence of cardiovascular death and heart failure in high-risk patients with cardiovascular disorders, presumably on account of the multifaceted effects of this drug class [5]. DPP-4 inhibitors facilitate insulin secretion via increasing the secretion of active glucagon-like peptide-1 (GLP-1), an incretin $[6,7]$. Therefore, the mechanism of the hypoglycemic action of SGLT2 inhibitors differs from that of the DPP-4 inhibitors. The present study was conducted to determine whether concurrent administration of a DPP-4 inhibitor, whose mechanism of action differs from that of the SGLT1 inhibitors, might yield further improvement of the glycemic control in Japanese type 2 diabetes mellitus patients showing relatively good glycemic control under SGLT2 inhibitor monotherapy.

\section{Materials and Methods}

\section{Subjects}

This is a retrospective non-randomized study. Each potential subject gave informed consent for participation in this study after receiving an explanation about the objective of the study from his/ her attending physician. This clinical trial was officially registered as an open-label study (ID: UMIN000021584).

Among the patients enrolled in this study, there were patients who were taking sulfonyl ureas and/or biguanides (hypoglycemic agents), lipid-lowering agents, and/or antihypertensive drugs before the start of the study. These patients continued to take those drugs at the same doses during the study period. Patients with an estimated glomerular filtration rate (eGFR) of less than 45 were excluded from the study. The primary endpoint of this study was the change in the level of HbA1c.

The study population consisted of 214 ambulatory Japanese patients with type 2 diabetes mellitus. The subjects were allocated to two groups, i.e., a group that received treatment with a SGLT2 inhibitor alone, or SGLT2 monotherapy ( $\mathrm{n}=18 ; 12$ men and 6 women; age: $54 \pm 12$ years), and a group that received combined SGLT2 inhibitor + DPP-4 inhibitor therapy ( $\mathrm{n}=196,145$ men and 51 women; age: $55 \pm 11$ years).

\section{Therapeutic regimens and administration}

The following five SGLT2 inhibitor preparations were used in this study: luseogliflozin, dapagliflozin, tofogliflozin, empagliflozin, and

- Table 1 Dose levels of drugs.

\begin{tabular}{|l|l|l|}
\hline \multirow{3}{*}{ SGLT2 inhibitor } & Name of drug & Dose level \\
\cline { 2 - 3 } & Luseogliflozin & $2.5 \mathrm{mg} /$ day \\
\cline { 2 - 3 } & Dapagliflozin & $5 \mathrm{mg} /$ day \\
\cline { 2 - 3 } & Tofogliflozin & $20 \mathrm{mg} /$ day \\
\cline { 2 - 3 } & Empagliflozin & $10 \mathrm{mg} /$ day \\
\cline { 2 - 3 } & Canagliflozin & $100 \mathrm{mg} /$ day \\
\hline DPP-4 inhibitor & Sitagliptin & $100 \mathrm{mg} /$ day \\
\cline { 2 - 3 } & Vildagliptin & $100 \mathrm{mg} /$ day \\
\cline { 2 - 3 } & Alogliptin & $25 \mathrm{mg} /$ day \\
\cline { 2 - 3 } & Anagliptin & $200 \mathrm{mg} /$ day \\
\cline { 2 - 3 } & Linagliptin & $5 \mathrm{mg} /$ day \\
\hline
\end{tabular}

canagliflozin. Five DPP-4 inhibitor preparations were also used, including sitagliptin, vildagliptin, alogliptin, anagliptin, and linagliptin. The SGLT2 inhibitors and DPP-4 inhibitors were administered once daily, before or after breakfast, for 6 months. The dose levels used of the drugs are shown in > Table 1. Each subject of the SGLT2 inhibitor monotherapy group received a SGLT2 inhibitor preparation. Each subject of the combined SGLT2 inhibitor + DPP-4 inhibitor group received a SGLT2 inhibitor preparation plus a DPP-4 inhibitor preparation.

\section{Measurements of the body weight, body-mass index (BMI), hemoglobin A1c (HbA1c), serum lipids and other biochemical parameters}

The body weight of the subjects was determined before the initiation of the study medication(s) (baseline) and after 6 months of treatment with the study medication(s). At the baseline and after 6 months of treatment with the study medication(s), random blood samples were obtained and the separated sera were stored frozen at $-80^{\circ} \mathrm{C}$ until the assays for the blood biochemical parameters. The Handa Medical Association Health Center (Aichi, Japan) was entrusted with measurement of the blood $\mathrm{HbA} 1 \mathrm{c}$ and serum lipid, aspartate aminotransferase (AST), alanine aminotransferase (ALT), $\gamma$-glutamyltransferase ( $\gamma$-GTP) and uric acid levels; the latter five parameters were analyzed in an autoanalyzer (JCA-BM8000 series, JAOL, Tokyo, Japan). The blood HbA1c level was determined by automated high-performance liquid chromatography (HPLC) (HLC723GX, Tosoh Corporation, Tokyo, Japan).

\section{Statistical analysis}

All the data are expressed as the mean \pm SD. The comparison of prevs. post-treatment data was conducted using the two-way ANOVA and Student's t-test. Statistical significance was set at $p<0.05$. Power analysis was performed using $G$ Power (Faul, Erdfelder, Lang \& Buchner).

\section{Results}

\section{SGLT2 inhibitor monotherapy}

The results are presented in $>$ Table 2 . The data concerning the SGLT2 inhibitors in this study represent the integrated data of patients treated with one of the 5 types of SGLT2 inhibitors. The blood HbA1c value tended to decrease following SGLT2 inhibitor treatment for 6 months, although the degree of decrease did not reach statistical significance. The body weight and BMI also decreased. The serum lipid profile tended to show improvement, although the improvement did not reach statistical significance. In addition to the above effects, the serum levels of AST, ALT, $\gamma$-GTP, and uric acid also decreased following the treatment.

\section{Combined SGLT2 inhibitor + DPP-4 inhibitor therapy}

The results are presented in $>$ Table 2 . The blood HbA1c value decreased in response to combined SGLT2 inhibitor + DPP-4 inhibitor therapy. The body weight and BMI also decreased. As for the serum lipid profile, the serum HDL-cholesterol (HDL-C) increased, whilst the serum LDL and total cholesterol also increased. The serum levels of AST, ALT, y-GTP and uric acid decreased. 
- Table 2 Responses to combined SGLT2 inhibitor+DPP4 inhibitor therapy.

\begin{tabular}{|c|c|c|c|c|}
\hline & \multicolumn{2}{|c|}{ SGLT2 inhibitor monotherapy } & \multicolumn{2}{|c|}{ Combined SGLT2 inhibitor + DPP4 inhibitor therapy } \\
\hline $\mathrm{n}$ & \multicolumn{2}{|c|}{$\mathrm{n}=18$ (male: 12 ; female: 6 ) } & \multicolumn{2}{|c|}{$\mathrm{n}=196$ (male: $145 ;$ female: 51 ) } \\
\hline Age (yrs.) & \multicolumn{2}{|c|}{$54 \pm 12$} & \multicolumn{2}{|c|}{$55 \pm 11$} \\
\hline \multirow[t]{2}{*}{ Duration of illness (yrs.) } & \multicolumn{2}{|c|}{$13 \pm 8$} & \multicolumn{2}{|c|}{$12 \pm 7$} \\
\hline & Baseline & 6 mo after & Baseline & 6 mo after \\
\hline Body weight $(\mathrm{kg})$ & $87.0 \pm 26.7$ & $85.4 \pm 26.1^{*}$ & $77.6 \pm 16.8$ & $75.5 \pm 16.6^{* *}$ \\
\hline BMI $\left(\mathrm{kg} / \mathrm{m}^{2}\right)$ & $30.2 \pm 6.2$ & $29.7 \pm 6.3^{*}$ & $28.1 \pm 5.3$ & $27.2 \pm 4.6^{* *}$ \\
\hline HbA1c (\%) & $7.4 \pm 1.0$ & $7.3 \pm 0.9$ & $7.1 \pm 0.9$ & $6.9 \pm 0.8^{* *}$ \\
\hline AST (U/L) & $33 \pm 18$ & $25 \pm 8^{*}$ & $26 \pm 17$ & $23 \pm 11 * *$ \\
\hline $\operatorname{ALT}(\mathrm{U} / \mathrm{L})$ & $36 \pm 20$ & $27 \pm 13^{*}$ & $32 \pm 26$ & $25 \pm 16^{* *}$ \\
\hline $\mathrm{Y}-\mathrm{GTP}(\mathrm{U} / \mathrm{L})$ & $37 \pm 24$ & $29 \pm 16^{*}$ & $42 \pm 46$ & $36 \pm 32 * *$ \\
\hline \multicolumn{5}{|l|}{ Uric acid $(\mathrm{mg} / \mathrm{dL})$} \\
\hline Male & $5.5 \pm 1.3$ & $5.1 \pm 1.4^{* *}$ & $5.5 \pm 1.2$ & $5.0 \pm 1.1^{* *}$ \\
\hline Female & $4.6 \pm 0.9$ & $3.9 \pm 1.3^{* *}$ & $4.8 \pm 1.3$ & $3.9 \pm 1.1^{* *}$ \\
\hline T-cho (mg/dL) & $175 \pm 31$ & $173 \pm 30$ & $176 \pm 29$ & $180 \pm 32 * *$ \\
\hline HDL-cho (mg/dL) & $57 \pm 16$ & $58 \pm 18$ & $58 \pm 14$ & $61 \pm 15^{* *}$ \\
\hline LDL-cho (mg/dL) & $97 \pm 31$ & $94 \pm 26$ & $97 \pm 23$ & $100 \pm 27^{*}$ \\
\hline Triglyceride (mg/dL) & $153 \pm 78$ & $133 \pm 92$ & $148 \pm 86$ & $142 \pm 95$ \\
\hline
\end{tabular}

\section{Comparison between SGLT2 inhibitor monotherapy and combined SGLT2 inhibitor + DPP-4 inhibitor therapy for individual DPP-4 inhibitors}

Patients receiving treatment with a SGLT2 inhibitor received concurrent administration of one of 5 different DPP-4 inhibitors, and the effects of the concurrent SGLT2 + DPP-4 inhibitor therapy were assessed by the type of DPP-4 inhibitor used; the results are summarized in $>$ Table 3. There was a significant decrease of the blood $\mathrm{HbA} 1 \mathrm{c}$ value in the patients receiving concurrent administration of sitagliptin (one of the DPP-4 inhibitors used) with the SGLT2 inhibitor, whereas the blood $\mathrm{HbA} 1 \mathrm{c}$ value only tended to decrease, the change not reaching statistical significance, in the patients receiving any of the other 4 DPP-4 inhibitors in combination with the SGLT2 inhibitor. In regard to the effect on the body weight and BMI, both these physical parameters decreased significantly, irrespective of the DPP-4 inhibitor preparation used in combination with the SGLT2 inhibitor. The serum AST, ALT and y-GTP levels (indicators of hepatic function) also improved significantly in the patients who received sitagliptin in combination with the SGLT2 inhibitor. In the patients who received vildagliptin concurrently with the SGLT2 inhibitor also, the serum AST and ALT levels decreased, whereas the serum $y$-GTP level showed no decrease. No decrease of the serum AST, ALT or $\gamma$-GTP was observed in the patients who received alogliptin or anagliptin in combination with the SGLT2 inhibitor. In the patients who received linagliptin with the SGLT2 inhibitor, the serum ALT and $y$-GTP levels decreased, whereas the serum AST level remained unchanged.

As for the serum uric acid level, the serum uric acid level was decreased in both male and female patients in the SGLT2 inhibitor monotherapy group. On the other hand, the uric acid was decreased both male and female patients in the combined SGLT2 inhibitor + sitagliptin, vildagliptin or alogliptin therapy. However, combined SGLT2 inhibitor + anagliptin decreased the uric acid level in male patients, and combined SGLT2 inhibitor + linagliptin decreased the uric acid level in female patients.

With regard to the impact on the serum lipid profile, elevation of the serum HDL-C was observed in the patients receiving concurrent administration of sitagliptin with a SGLT2 inhibitor. Concomitant use of vildagliptin, alogliptin, or anagliptin with the SGLT2 inhibitor also caused elevation of the serum HDL-C, while no such elevation was seen in the patients receiving linagliptin, as the DPP-4 inhibitor, with the SGLT2 inhibitor. Concurrent administration of a DPP-4 inhibitor, regardless of the preparation used, with a SGLT2 inhibitor had no effect on the serum total cholesterol, LDL-cholesterol (LDL-C) or triglyceride level.

\section{Discussion}

Concurrent use of two or more hypoglycemic agents with differing mechanisms of action may be useful in type 2 diabetes mellitus patients showing inadequate glycemic control under treatment with a single drug. It is considered that drugs whose blood-sugar-lowering mechanism does not involve the pancreatic beta cells may be more effective for concurrent administration, especially in view of the lower risk of hypoglycemia.

It has been reported that SGLT2 inhibitors exert their hypoglycemic effect by facilitating urinary excretion of excessive circulating glucose [3]. This class of drugs cause selective inhibition of SGLT2 [1,2], a transporter protein that mediates reabsorption of about $90 \%$ of the glucose filtered through the glomeruli. In addition to exerting a hypoglycemic effect, SGLT2 inhibitors have also been reported to reduce the body weight, lower the blood pressure, and improve lipid metabolism and uric acid metabolism [4]. In one placebo-controlled study conducted in high-risk patients with cardiovascular disorders, treatment with a SGLT2 inhibitor, as compared to placebo, significantly suppressed the incidence of car- 


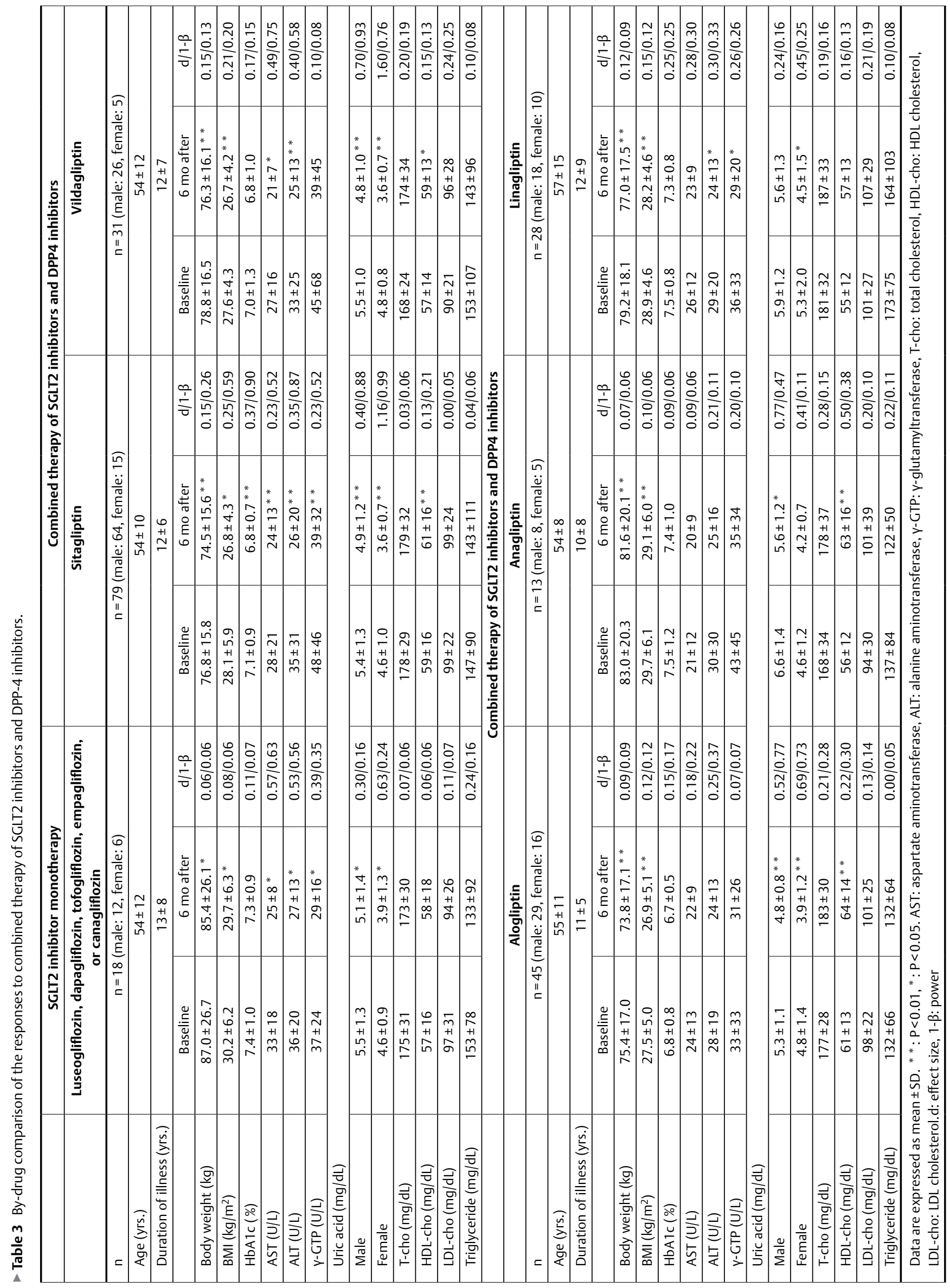


diovascular death and heart failure, presumably on account of the versatile effects of this class of drugs [5].

DPP-4 inhibitors have been shown to facilitate insulin secretion via non-pancreatic beta cell-mediated increase of active GLP-1, an incretin, and it has been suggested that inhibition of DPP-4 may also afford extra-pancreatic effects in addition to the effect on the blood glucose level $[8,9]$.

In general, many treatment options exist for patients with poor glycemic control with markedly elevated blood HbA1c values, because those patients are scarcely at risk of developing hypoglycemic episodes. On the other hand, in patients with relatively good glycemic control, it is important to select a treatment agent that would not entail the risk of development of hypoglycemia. How to safely reduce the blood glucose level to an HbA1c of $6.9 \%$ level without increasing the risk of development of diabetic complications is an important issue in such patients. In view of this, we conducted a trial of combined SGLT2 inhibitor + DPP-4 inhibitor treatment, both classes of drugs being generally thought as being unlikely to cause hypoglycemic attacks, in patients with type 2 diabetes mellitus showing relatively good glycemic control, using various combinations of SGLT2 inhibitors and DPP4 inhibitors, to explore which drug combinations might be more effective. Five different SGLT2 inhibitor preparations were used in this study, in which the therapeutic response data from patients receiving SGLT2 inhibitor therapy alone were integrated into SGLT2 inhibitor monotherapy group data for analysis, without distinction among the preparations prescribed. On the other hand, 5 different DPP- 4 inhibitor preparations were also used, and these were dealt with individually for comparison with the SGLT2 inhibitor monotherapy group.

First, comparison of the therapeutic responses between the integrated SGLT2 inhibitor monotherapy group and combined SGLT2 inhibitor + DPP-4 inhibitor therapy group is presented in > Table 2 . As can be seen, no significant decrease of the blood HbA1c value was observed in the SGLT2 inhibitor monotherapy group, while significant reduction of this parameter was noted in the combined therapy group, indicating the favorable effect of concurrent administration of a DPP-4 inhibitor with a SGLT2 inhibitor. The serum $\mathrm{HDL}-\mathrm{C}$ level increased in the combined drug therapy group, again indicating a favorable effect of the combined therapy. The body weight, BMI and serum uric acid level are widely recognized to decrease in response to treatment with a SGLT2 inhibitor alone, and our present study yielded consistent results. These effects were maintained even after the addition of a DPP- 4 inhibitor. The percent decreases of the 3 parameters were comparable between the two groups, and were not enhanced by combined administration of a DPP-4 inhibitor with the SGLT2 inhibitor.

Kusunoki et al. have reported that treatment with a SGLT2 inhibitor is associated with improvement of the hepatic function parameters, namely, serum AST, ALT and $\mathrm{y}$-GTP [10]. Consistent with this report, in the present study, treatment with the SGLT2 inhibitor alone was associated with a lowering of the serum levels of AST, ALT and $y$-GTP. Furthermore, this effect was maintained even after the addition of a DPP-4 inhibitor to the SGTL2 inhibitor therapy. The percent decreases in these 3 parameters were comparable between the two groups and were not enhanced by the addition of the DPP-4 inhibitor to the treatment regimen.
In this study, we examined the correlations between the percent changes of the $\mathrm{HbA} 1 \mathrm{C}$ values and percent changes of the serum AST, ALT and $\gamma$-GTP, and obtained the following results: the correlation coefficients ( $r$ values) between the percent changes of the HbA1c levels and percent changes of the serum AST, serum ALT and serum $\mathrm{Y}$-GTP levels were $0.60(P<0.05), 0.55(P<0.05)$ and 0.53 $(P<0.05)$, respectively, in the SGLT2 inhibitor monotherapy group. In the combined SGLT2 inhibitor plus DPP4 inhibitor group, the corresponding values of the correlation coefficient $(r)$ were 0.31 $(P<0.01), 0.33(P<0.05)$ and $0.47(P<0.01)$, respectively. These results indicate that the improved glycemic control was also associated with improvement in the markers of liver dysfunction.

As for effects on the serum lipid profile, treatment with a SGTL2 inhibitor alone had no influence on the serum lipid profile. In the combined SGLT2 inhibitor + DPP-4 inhibitor therapy group, on the other hand, elevation of the serum HDL-C was observed, indicating the favorable effect of addition of the DPP-4 inhibitor. However, the serum total cholesterol and LDL-C also increased.

Secondly, the therapeutic responses to combined SGLT2 inhibitor + DPP-4 inhibitor therapy were assessed for the 5 individual DPP-4 preparations used, and the results are summarized in $>$ Table 3 . Of the five DPP- 4 inhibitors used, only sitagliptin led to significant decrease of the $\mathrm{HbA} 1 \mathrm{c}$, one of the most important indicators of blood glucose control, when administered in combination with a SGLT2 inhibitor in the combined SGLT2 + DPP-4 inhibitor therapy group. On the other hand, only a tendency towards decrease of the HbA1c was observed for the remaining 4 DPP-4 inhibitors administered in combination with a SGLT2 inhibitor.

Elevation of the serum HDL-C without elevation of the serum LDL-C or total cholesterol was observed when sitagliptin was used in the combined treatment group. This combination treatment also resulted in lowering of the body weight, BMI, and serum levels of AST, ALT, $Y$-GTP and uric acid, the percent decrease of each of these being essentially the same as that in the SGLT2 inhibitor monotherapy group. There was no consistent trend of the effects for any of the other four DPP-4 inhibitors administered in combination with a SGLT2 inhibitor.

It was first pointed out by Merovci et al. that SGLT2 inhibitor therapy with concomitant use of an insulin-secretion-facilitating DPP-4 inhibitor would be effective in the treatment of diabetes mellitus, since SGLT2 inhibitors promote glucagon secretion to increase endogenous glucose production [11].

There are reports suggesting the greater $\mathrm{HbA} 1 \mathrm{c}-$ lowering potency of combined SGLT2 inhibitor + DPP-4 inhibitor therapy as compared to monotherapy with either a SGLT2 inhibitor or a DPP-4 inhibitor $[12,13]$. The baseline blood $\mathrm{HbA1}$ c values of the subjects in these studies were, however, 8-9\%, indicating poor glycemic control.

Regarding the uric acid, it is generally recognized that genderrelated differences exist in the serum uric acid levels. In view of this, we analyzed the serum uric acid data in the present series separately for male patients and female patients. Specifically, the baseline serum uric acid level was significantly higher in the male than in the female patients in both the SGLT2 inhibitor monotherapy and combined SGLT2 inhibitor plus DPP4 inhibitor groups $(P<0.05)$. In the SGLT2 inhibitor monotherapy group, the serum uric acid level decreased in both male and female patients. In the combined SGLT2 
inhibitor plus DPP4 inhibitor (sitagliptin, vidagliptin or alogliptin) group, and the serum uric acid levels significantly decreased in both male and female patients. On the other hand, combined SGLT2 inhibitor + anagliptin significantly decreased the uric acid level in male patients, and combined SGLT2 inhibitor + linagliptin significantly decreased the uric acid level in female patients.

The present study results confirm that the serum uric acid levels decreased in response to both the study treatments in male and/ or female patients. In regard to the mechanism underlying such reduction of the uric acid level, it has been considered that the high urinary glucose level resulting from SGLT2 inhibition suppresses the reabsorption of uric acid into the blood via GLUT9 isoform 2 (SLC2A9) in the proximal tubule, promoting excretion of uric acid into the urine [14].

The mean HbA1c values in the subjects included in this study were in the range of $6.8-7.5 \%$, suggesting that the patients showed relatively good control of the blood glucose levels.

Our present findings suggest that combined SGLT2 inhibitor + DPP-4 inhibitor therapy has a rather modest additive or synergistic effect in patients with relatively good glycemic control with blood HbA1c values of around $7 \%$. In terms of the effects on the glucose parameter, i. e., HbA1c values, concurrent administration of sitagliptin, as compared to the other 4 DPP-4 inhibitors, with a SGLT2 inhibitor yielded the best results. However, since the number of study subjects was different in the groups, therefor we performed power analysis. The result of the power analysis indicated that effect size (d) and power (1- $\beta$ ) in the SGLT2 inhibitor plus sitagliptin were greater than other combined SGLT2 inhibitor + DPP4 inhibitor. The analysis showed that the combined SGLT2 inhibitor plus sitagliptin regimen was superior in efficacy to any of the other four treatment regimens examined in this study.

This was a retrospective study. Therefore, no particular criteria were set for the grouping. There was no significant inter-group difference in the pre-treatment baseline body weight or $\mathrm{HbA} 1 \mathrm{c}$ level. The limitations of this study were: (1) its non-randomized, and (2) its small sample size. We plan to conduct a larger-scale study in the near future.

\section{Conclusion}

The results of individual assessment of the effects of five DPP-4 inhibitors administered in combination with a SGLT2 inhibitor in type 2 diabetes mellitus patients showing relatively good glycemic control under treatment with a SGLT2 inhibitor revealed that concurrent use of sitagliptin in combination with a SGLT2 inhibitor yielded the best results, both in terms of the effects on the blood glucose and in terms of the effect on the serum lipid parameters.

\section{Conflict of Interest}

The authors report no conflicts of interest.

\section{References}

[1] Wright EM. Glucose transport families SLC5 and SLC50. Mol Aspects Med 2013; 34: 183-196

[2] Wright EM, Loo DD, Hirayama BA. Biology of human sodium glucose transporters. Physiol Rev 2011; 91: 733-794

[3] DeFronzo RA, Davidson JA, Del Prato S. The role of the kidneys in glucose homeostasis: a new path towards normalizing glycaemia. Diabetes Obes Metab 2012; 14: 5-14

[4] Seino Y, Sasaki T, Fukatsu A et al. Efficacy and safety of luseogliflozin as monotherapy in Japanese patients with type 2 diabetes mellitus: A randomized, double-blind, placebo-controlled, phase 3 study. Curr Med Res Opin 2014; 30: 1245-1255

[5] Zinman B, Wanner C, Lachin JM et al. EMPA-REG OUTCOME Investigators. Empagliflozin, cardiovascular outcomes, and mortality in type 2 diabetes. N Engl J Med 2015; 373: 2117-2128

[6] Kim YG, Hahn S, Oh T] et al. Differences in the glucose-lowering efficacy of dipeptidyl peptidase- 4 inhibitors between Asians and non-Asians: A systematic review and meta-analysis. Diabetologia 2013; 56: 696-708

[7] Esposito K, Cozzolino D, Bellastella G et al. Dipeptidyl peptidase-4 inhibitors and $\mathrm{HbA} 1 \mathrm{c}$ target of $<7 \%$ in type 2 diabetes: meta-analysis of randomized controlled trials. Diabetes Obes Metab 2011; 13 : 594-603

[8] Shah Z, Kampfrath T, Deiuliis JA et al. Long-term dipeptidyl-peptidase 4 inhibition reduces atherosclerosis and inflammation via effects on monocyte recruitment and chemotaxis. Circulation 2011; 124 : 2338-2349

[9] Monami M, Lamanna C, Desideri CM et al. DPP-4 inhibitors and lipids: Systematic review and meta-analysis. Adv Ther 2012; 29: 14-25

[10] Kusunoki M, Sato D, Natsume Y et al. Luseogliflozin, a sodium glucose co-transportet 2 inhibitor, alleviates hepatic impairment in Japanese patients with type 2 diabetes mellitus. Drug Res 2016; 66: 603-606

[11] Merovci A, Solis-Herrera C, Daniele G et al. Dapagliflozin improves muscle insulin sensitivity but enhances endogenous glucose production. J Clin Invest 2014; 124: 509-514

[12] Rosenstock J, Hansen L, Zee P et al. Dual add-on therapy in type 2 diabetes poorly controlled with metformin monotherapy: A randomized double-blind trial of saxagliptin plus dapagliflozin addition versus single addition of saxagliptin or dapagliflozin to metformin. Diabetes Care 2015; 38: 376-383

[13] Scheen AJ. DPP4 inhibitor plus SGLT-2 inhibitor as combination therapy for type 2 diabetes: from rationale to clinical aspects. Expert Opin Drug Metab Toxicol 2016; 12: 1407-1417

[14] Chiho Y, Samukawa Y, Sakai S et al. SGLT-2 inhibitor lowers serum uric acid through alteration of uric acid transport activity in renal tubule by increased glycosuria. Biopharm Drug Dispos 2014; 35: 391-404 